\title{
Endoscopic ultrasound-guided rendezvous recanalization of a completely obstructed esophagogastric anastomosis
}

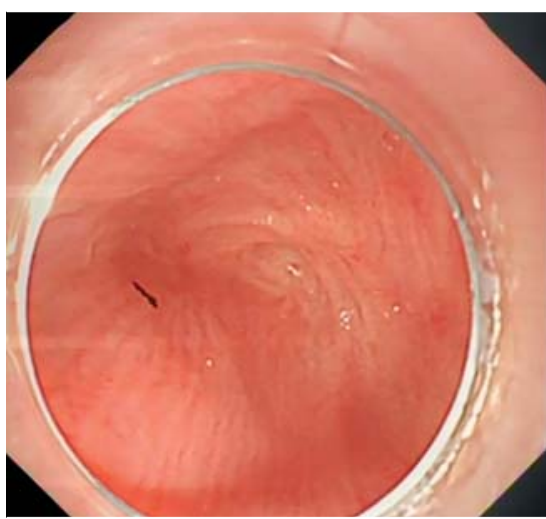

- Fig. 1 Completely obstructed esophagogastric anastomosis in a 63-year-old woman.

A 63-year-old woman who had undergone resection of adenocarcinoma at the gastroesophageal junction was admitted with complete obstruction of the esophagogastric anastomosis ( $\mathbf{F i g . 1}$ ). For the past 2 years, gastrostomy tube feeding had been her only source of enteral nutrition. Endoscopic ultrasound (EUS)guided rendezvous recanalization of the obstruction was attempted to allow her to resume oral intake ( $\triangleright$ Video 1 ).

An antegrade linear ultrasound endoscope was placed at the blind end of the esophagus to locate the gastric lumen, determine the optimal puncture site, and guide the puncture process. A retrograde standard endoscope with biopsy forceps was introduced via the gastrostomy tube to offer direct vision of the gastric lumen, assist puncture, and function as a location sign with a strong echo (\$Fig.2). A 19-gauge needle was advanced towards the gastric lumen under Doppler EUS guidance (> Fig.3). Once the needle had passed beyond the obstruction, a guidewire was inserted through it. Next, a 6-Fr biliary dilation catheter was advanced over the guidewire. Sequential endoscopic balloon dilations were then performed over a 2-month period before a fully covered

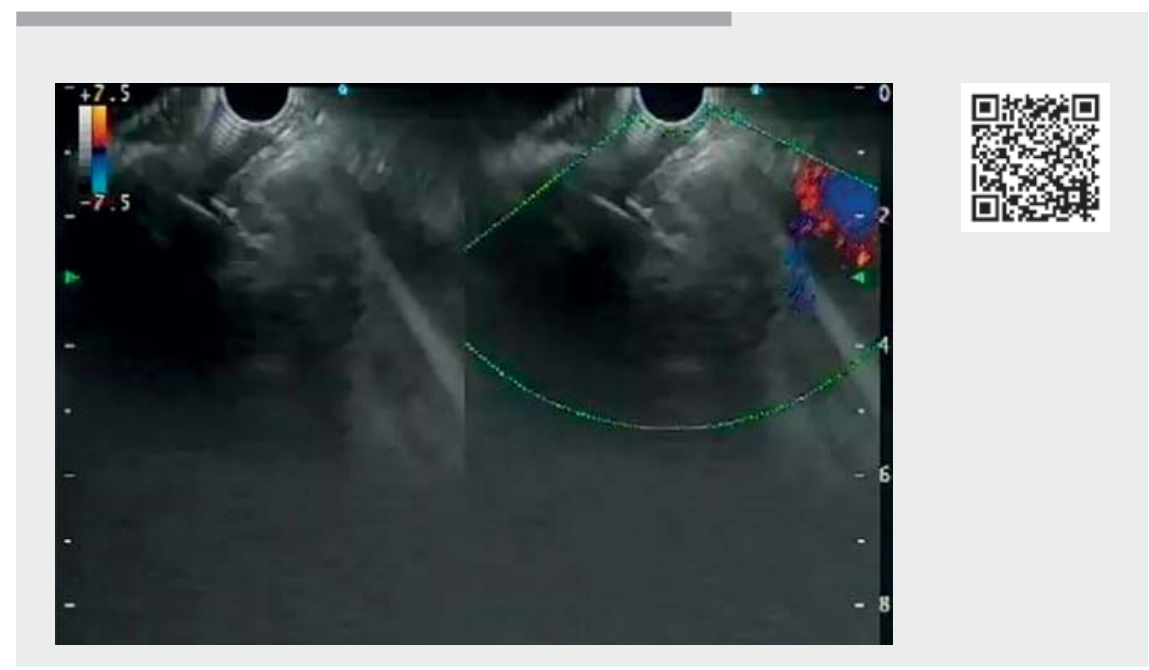

$\checkmark$ Video 1 Endoscopic ultrasound-guided rendezvous recanalization of a completely obstructed esophagogastric anastomosis.

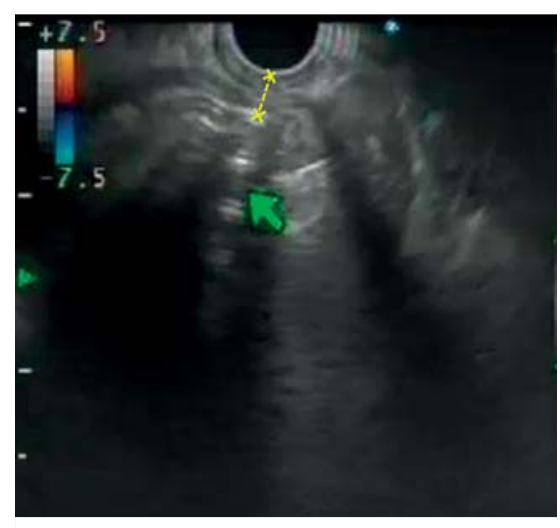

- Fig. 2 The endoscope with forceps (green arrow) in the gastric lumen was detected by endoscopic ultrasound (EUS) as a strong echo, from the tip of which the puncture route was marked (yellow dotted line)

retrievable metal stent $(60 \times 20 \mathrm{~mm})$ was deployed ( Fig.4). No adverse event occurred. The patient started to eat semiliquid food and gained weight, so the gastrostomy tube was removed. After 2 months, the esophageal stent was retrieved, followed by another dilation, and the tract from the esophagus to the

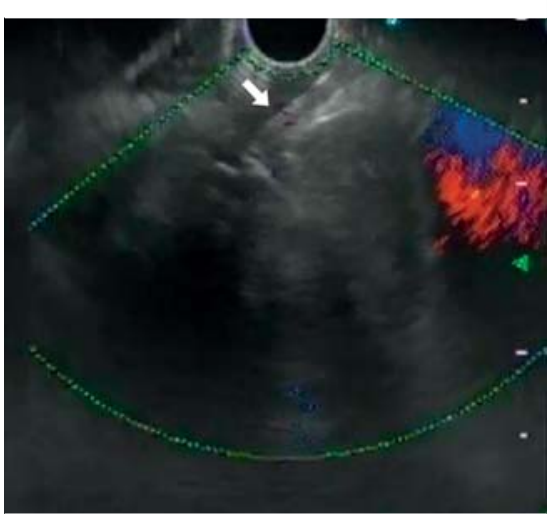

Fig. 3 A 19-gauge needle (white arrow) was advanced towards the gastric cavity under Doppler EUS guidance.

stomach was seen to be well formed ( Fig. 5).

The treatment of a completely obstructed esophagogastric anastomosis was often reported as part of the treatment of complete esophageal obstruction (CEO), and combined antegrade-retrograde endoscopic dilation (CARD) has been the most widely used approach in CEO [1, 2]. However, the fluoroscopically guided access procedure is not precise 


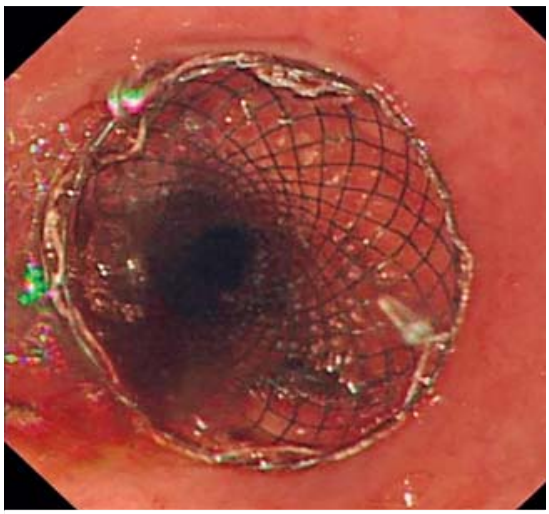

- Fig. 4 A fully covered retrievable metal stent $(60 \times 20 \mathrm{~mm})$ was placed.

enough because of limited real-time guidance and the invisibility of adjacent structures and vessels; this is why we introduced EUS into the scenario. Although we report it here in the setting of anastomotic obstruction, the technique may be extrapolated to the treatment of CEO from other causes.

Endoscopy_UCTN_Code_TTT_1AO_2AN

\section{Funding}

1.3.5 Project for Disciplines of Excellence Clinical Research Incubation Project, West China Hospital, Sichuan University 20HXFH016

\section{Competing interests}

The authors declare that they have no conflict of interest.

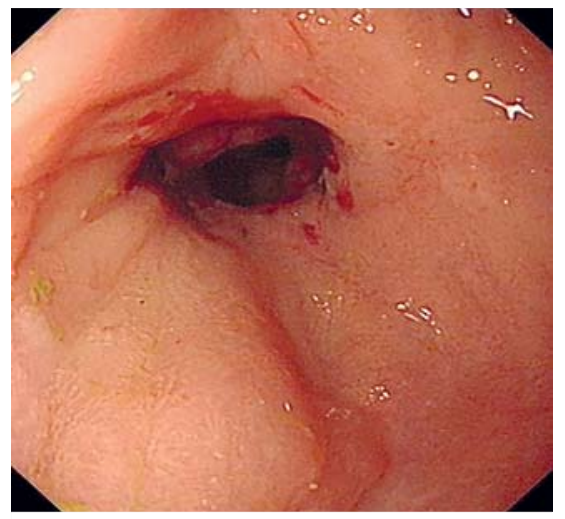

- Fig. 5 The stent was removed, followed by another dilation, and the tract from the esophagus to the stomach was seen to be well formed.

The authors

Yu-Yan Zhang ", Lian-Song Ye ", Yi Mou, ChunCheng Wu, Qing-Hua Tan, Ke Liao, Bing Hu ${ }^{\odot}$ Department of Gastroenterology, West China Hospital, Sichuan University, Chengdu, Sichuan, China

\section{Corresponding author}

\section{Bing Hu, MD}

Department of Gastroenterology, West China Hospital of Sichuan University, No. 37 Guo Xue Alley, Wu Hou District, Chengdu 610041, Sichuan, P. R. China

hubingnj@163.com

\section{References}

[1] Jayaraj M, Mohan BP, Mashiana $\mathrm{H}$ et al. Safety and efficacy of combined antegrade and retrograde endoscopic dilation for complete esophageal obstruction: a systematic review and meta-analysis. Ann Gastroenterol 2019; 32: 361-369

[2] Perbtani Y, Suarez A, Wagh M. Emerging techniques and efficacy of endoscopic esophageal reconstruction and lumen restoration for complete esophageal obstruction. Endosc Int Open 2016; 4: E136-E142

\section{Bibliography}

Endoscopy 2022; 54: E137-E138

DOI 10.1055/a-1443-3943

ISSN 0013-726X

published online 20.4.2021

(c) 2021. Thieme. All rights reserved.

Georg Thieme Verlag KG, Rüdigerstraße 14, 70469 Stuttgart, Germany

\section{ENDOSCOPY E-VIDEOS}

https://eref.thieme.de/e-videos

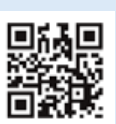

Endoscopy E-Videos is an open access online section, reporting on interesting cases and new techniques in gastroenterological endoscopy. All papers include a high quality video and all contributions are freely accessible online. Processing charges apply (currently EUR 375), discounts and wavers acc. to HINARI are available.

This section has its own submission website at

https://mc.manuscriptcentral.com/e-videos

* These authors contributed equally. 